\title{
Efficacy of gentamicin $0.3 \%$ solution of oral erosions healing in patients with severe generalized recessive dystrophic epidermolysis bullosa and its impact on the expression of type VII collagen
}

\author{
Katarzyna Osipowicz ${ }^{1}$, Piotr Wychowanski², Pawel Nieckula², Sara Shamsa ${ }^{3}$, Katarzyna Wertheim-Tysarowska ${ }^{4}$, \\ Katarzyna Wozniak ${ }^{1}$, Cezary Kowalewski ${ }^{1}$
}

\begin{abstract}
${ }^{1}$ Department of Dermatology and Immunodermatology, Medical University of Warsaw, Warsaw, Poland ${ }^{2}$ Department of Department of Oral Surgery, Medical University of Warsaw, Warsaw, Poland ${ }^{3}$ Department of Paediatric Dentistry, Medical University of Warsaw, Warsaw, Poland ${ }^{4}$ Department of Medical Genetics, Institute of Mother and Child, Warsaw, Poland
\end{abstract}

Adv Dermatol Allergol 2021; XXXVIII (6): 979-984 DOI: https://doi.org/10.5114/ada.2020.97072

\begin{abstract}
Introduction: Epidermolysis bullosa (EB) is a rare genetic skin disorder inherited either in autosomal recessive $(A R)$ or autosomal dominant (AD) manner and characterized by blistering of the skin and mucous membranes. According to a subtype of EB, the oral manifestations and dental involvement vary in frequency and in severity. The most severe dental problems occur in patients with junctional epidermolysis bullosa (JEB) and severe generalized dystrophic epidermolysis bullosa (RDEB) and involve enamel erosion and development of blisters followed by painful oral wounds. Oral mucosa lesions decrease patients' quality of life and may contribute to difficulties in nutrition leading to cachexia.

Aim: Assessment of efficacy of gentamicin $0.3 \%$ solution in the healing and preventing of oral erosions in patients with RDEB and evaluating its impact on the expression of type VII collagen.

Material and methods: The study included four female patients with RDEB, aged 16-42 who show different mutations in the COL7A1 gene and were administered the mouth rinse two times daily with a solution of $0.3 \%$ gentamycin for 4 consecutive weeks. Prior to and at the end of the study, the samples from oral mucosa were collected to estimate the expression of type VII collagen by immunofluorescence test.

Results: The clinical improvement of oral wounds healing and reduced number of new blisters and mucous membrane soreness as well as partial re-expression of type VII collagen was observed in all studied patients.

Conclusions: Topical gentamicin therapy of oral cavity in RDEB patients resulted in clinical improvement of mucosal lesions and re-expression of collagen type VII.
\end{abstract}

Key words: gentamycin $0.3 \%$ solution, VII collagen, oral mucosa, junctional epidermolysis bullosa, recessive dystrophic epidermolysis bullosa.

\section{Introduction}

Epidermolysis bullosa (EB) is a group of rare genetic skin disorders inherited either in autosomal recessive (AR) or autosomal dominant manner (AD). This genodermatosis is characterized by blistering of the skin and mucous membranes, most commonly related to mechanical trauma but also occurring spontaneously. The most severe dental problems occur in patients with junctional epidermolysis bullosa (JEB) and severe generalized dys- trophic epidermolysis bullosa (RDEB) and involve enamel erosion and development of blisters followed by painful oral wounds.

The development of oral erosions significantly decrease patients' quality of life and may contribute to difficulties in nutrition leading to cachexia. Oral manifestations and dental involvement in the course of EB vary in frequency and in severity according to the subtype [1]. In the EB simplex, the teeth are not affected; however, the

Address for correspondence: Prof. Cezary Kowalewski MD, PhD, Department of Dermatology and Immunodermatology, Medical University of Warsaw, Poland, phone: +48 506325 539, e-mail: ckowalewski@wum.edu.pl Received: 27.04.2020, accepted: 12.06.2020. 
JEB and RDEB types often display malformed teeth due to enamel hypoplasia, early caries development and gingival inflammation due to plaque accumulation [2] leading to the periodontal disease and tooth decay, due to underdevelopment of enamel [3]. The main oropharyngeal lesions are represented by vesiculo-bullous lesions, other types of lesions such as erythema, erosion and atrophy, may involve one or more of the 13 oropharyngeal mucosal sites (upper and lower lip, right and left buccal mucosa, upper and lower gingiva, upper and lower vestibule, hard palate, soft palate, oropharynx, floor of the mouth and tongue) with a variable frequency and severity [4]. Patients may develop microstomia and loss of tissue mobility within the lips and perioral tissues.

As some of the genes involved in the pathogenesis of the JEB subtypes (i.e. beta chain of laminin-332 and type 17 collagen) also play a pivotal role in the formation of normal teeth, the enamel may be affected from generalized pitting to generalized hypoplasia [4]. Heterozygous carriers of COL17A1 mutations have been shown to have enamel defects that range from horizontal hypoplastic bands to white mottled enamel [5]. In some individuals with JEB, the enamel hypoplasia may lead to a very thin layer of enamel on the tooth surface [6].

RDEB is due to pathogenic either homozygous or compound heterozygous mutation in the gene COL7A1 encoding collagen type VII resulting in the lack of or significant reduction in expression of this protein. Most RDEB patients present severe non-healing and painful oral wounds and limited ability to open mouth (ankyloglossia). It may result in reduced depth of the oral vestibule.

Gentamicin is one of the topical medications shown to be effective in the treatment of skin wounds in EB due to both its antiseptic activity and repairing properties of nonsense mutation, allowing for the read-through translation of the protein and the synthesis of a full-length protein [3, 7-9]. To the best of our knowledge, we are the first to propose topical treatment with gentamicin of mucosal erosions in patients with RDEB.

\section{Aim}

The aim of the study was to assess the clinical efficacy of gentamicin $0.3 \%$ solution in the treatment and preventing of oral mucosal lesions in 4 patients with RDEB and evaluate its impact on the expression of type VII collagen.

\section{Material and methods}

The study included four female patients, aged 16-42, selected from patients treated in the Department of Dermatology and Immunodermatology of the Medical University of Warsaw after obtaining the consent of the
Bioethics Committee of the Medical University of Warsaw (no. KB/218/2016).

Patients who displayed different mutations in the COL7A1 gene (Table 1) were administered the mouth rinse two times daily for 4 consecutive weeks. The formula was the following: (Rp. gentamycini 0.3, aqua dest, glicerinii aa ad 100.0).

The patients have to take one large spoonful of mouth wash $(10 \mathrm{ml})$, then rinse the mouth for $3 \mathrm{~min}$ and then split out. We have recommended our patient to abitain from drinks and meals for 30 min after the use of mouth rinse.

The clinical examinations were conducted every week. After 4 weeks patients had follow-up visits once a month.

Prior to and at the end of the study, the samples from oral mucosa were taken under local anaesthesia and collected for immunofluorescence analysis to estimate the expression of type VII collagen.

The samples were embedded in OCT compound at a low temperature and cut into sections of $6 \mu \mathrm{m}$ using a cryostat and tested with the use of monoclonal antibodies directed to a fragment of NC1 domain of type VII collagen (clone 7.2, Sigma) and human anti basement membrane zone antibodies from a patient with epidermolysis bullosa acquisita (EBA).

Monoclonal and polyclonal antibodies were visualized with a mix of goat anti-mouse IgG conjugated with Cy5 (Sigma) and goat anti-human IgG conjugated with FITC (Kappel). Primary and secondary antibodies were incubated with tissue for 30 min, then washed with PBS ( $2 \times 10 \mathrm{~min})$ and immersed in glycerol and observed under BioRad2000 confocal microscope.

\section{Results}

The treatment with gentamicin $0.3 \%$ solution in EB patients led to the significant improvement of oral wounds in all four patients (Figure 1). Noteworthy is that the number of new blisters/erosions was reduced at least three times, the surface area of erosions was reduced at least two times as well as reduction in mucous membrane soreness was observed. Additionally, the patients reported reduced frequency and intensity of bleeding gums when brushing teeth and other oral hygiene procedures. Stability of gums encouraged the patients to a more careful and accurate hygiene procedure of teeth and oral cavity. This phenomenon resulted in the significant decrease in the Plaque Index and Gingival Bleeding Index. Decreased halitosis was also noticed based on consistent opinions of patients, their doctors and family members.

The immunofluorescence study in 2 patients (patient no. 1 with severe heterozygous mutations c.682+1G>A/ c. $5532+6 \mathrm{~T}>\mathrm{C}$ and patient no. 2 with homozygous mutation c.425A>G), showed the lack of type VII collagen expression of both non-collagenous and collagenous part 

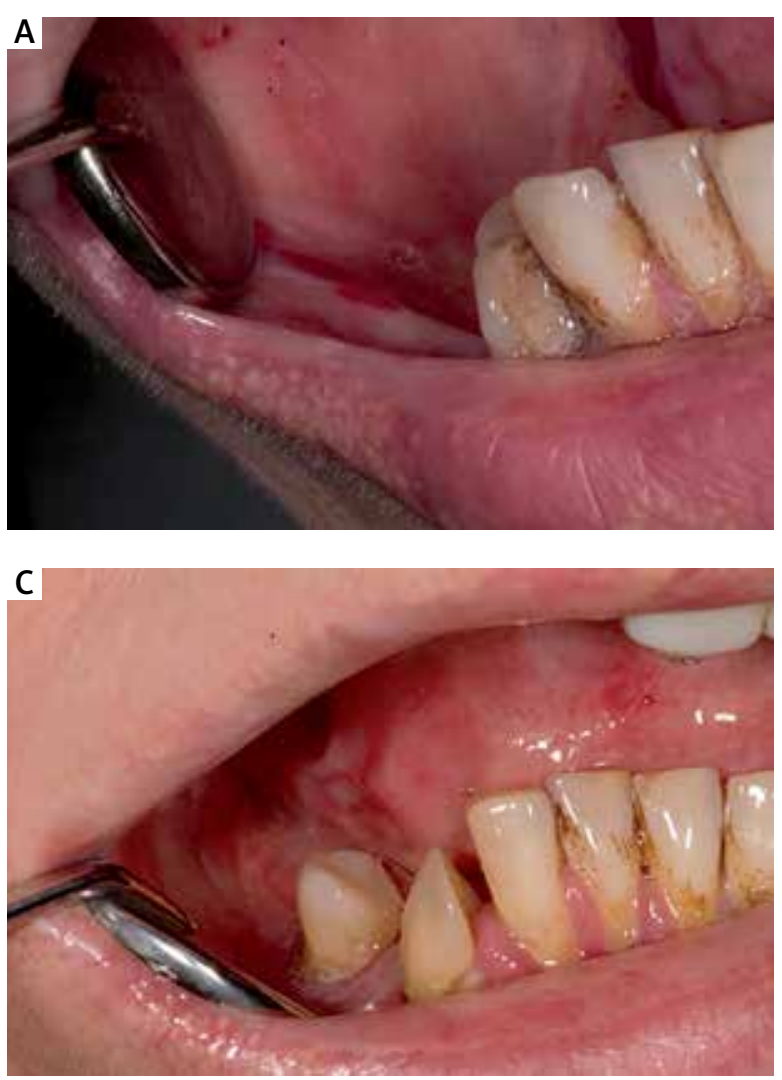

prior to the treatment and the weak expression of its collagenous part after the therapy.

Patients no. 3 and 4 with compound heterozygous mutations (c.425A>G/c.7904G >A and c.425A>G/ c.5884A $>C$, respectively) with a weak expression of collagen type VII before treatment presented an increased expression of both non-collagenous and collagenous part of collagen type VII after the therapy (Figure 2, Table 1).

\section{Discussion}

Recent studies have confirmed that in patients with RDEB, both topical application and intradermal injection of gentamicin to the skin erosions induced wound healing and production of type VII collagen and anchoring fibrils at the dermo-epidermal junction. Therapeutic effect after the intravenous injection of gentamicin lasts about three months from the treatment conclusion [10], which stays in agreement with the knowledge of the physiological degradation of type VII collagen in the skin [7]. It was demonstrated in the cell culture model of keratinocytes sampled from patients with $\mathrm{H}$-JEB that gentamicin induced the production of chain $\beta$ that composed laminin 332 and, in consequence, led to restored synthesis of all three laminin 332 chains $(\alpha, \beta, \gamma)$. In addition, in the gentamicin-stimulated cells, the adhesive function was restored to molecules binding to laminin 332, such as integrin $\alpha 6 \beta 4$. The restored synthesis of laminin 332 cor-

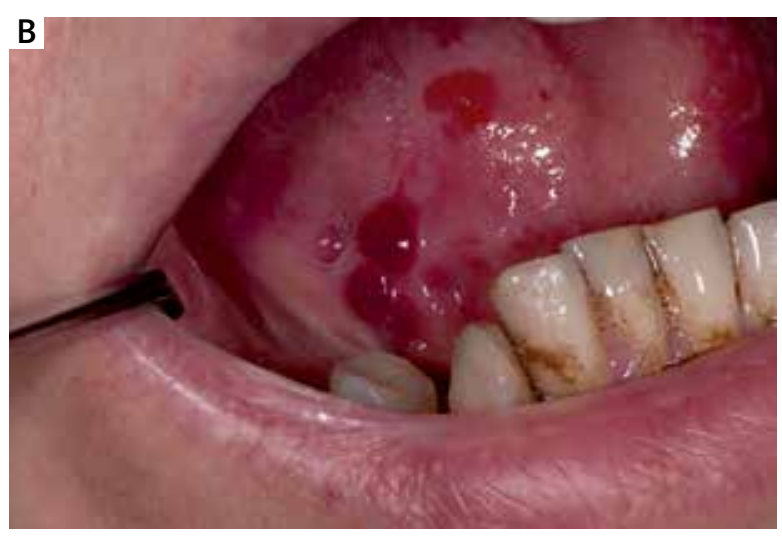

Figure 1. Photographic image of oral mucosa in the RDEB patient: $\mathbf{A}$ - lesions of the right cheek mucosa before administration of mouth rinse, $\mathrm{B}$ - lesions of the tongue before administration of mouth rinse, $\mathbf{C}$ - Healing of lesions after 4 weeks of mouth rinse application

rected the phenotype of $\mathrm{H}$-JEB cells by reversing aberrant cell morphology, low potential for growth, weak cell and substrate adhesion [11].

Divergent results of gentamicin treatment experienced by different researchers are explained by the latest studies demonstrating that mostly B1 component of gentamicin are able to correct genetic disorders by the PTC readthrough [12]. Gentamicin, used in medicine as an antibiotic, is a combination of several aminoglycosides and the content of the B1 component in the commercially available gentamicin is highly variable. Commercially available preparation of gentamicin is a composition of related aminoglycosides isolated from Micromonospora purpurea fermentation. It is composed mainly of major gentamicin components (C1, C1a, C2, $\mathrm{C} 2 \mathrm{a}$ and $(2 \mathrm{~b})$ and from related minor aminoglycosides [3]. It should be stressed that various pharmaceutical companies and various pharmacies use different sources of gentamicin. Normally, only the concentration of the three main components with the strongest antibacterial effect is determined, but the concentration of the $\mathrm{B} 1$ component is not. It is known from experimental research that $\mathrm{B} 1$ component is just a small additive to the drug content (2-5\%). It means that different pharmacies, while correctly filling the same prescription, can dispense a drug with varying content of the B1 component and therefore of varying efficacy, even for the same patient with RDEB. Therefore, in the future the concen- 

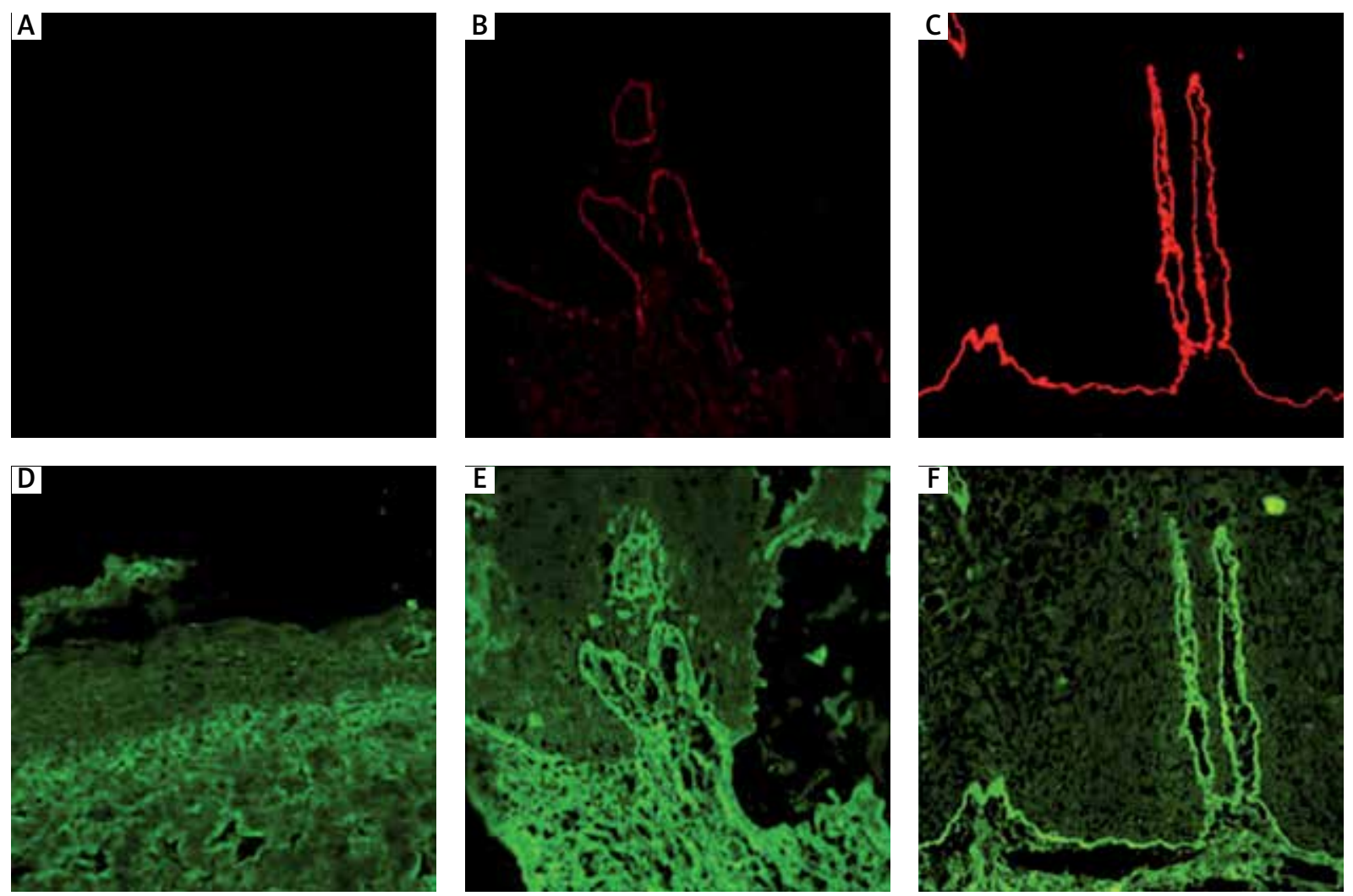

Figure 2. Immunofluorescence study showed partial re-expression of collagen VII in RDEB after treatment. Expression of amino terminal and non-collagenous domain - NC1 (red): A - before treatment, B - after treatment, C - healthy control. Expression of the collagenous part of collagen VII (green): D - before treatment, E - after treatment, $\mathbf{F}$ - healthy control

Table 1. RDEB patients included in the study and effectiveness of topical gentamicin treatment for restoration of collagen VII

\begin{tabular}{|c|c|c|c|c|c|c|c|}
\hline \multirow[t]{2}{*}{ No. } & \multirow[t]{2}{*}{ Sex } & \multirow[t]{2}{*}{ Age } & \multirow{2}{*}{$\begin{array}{c}\text { RDEB } \\
\text { Mutation in the gene COL7A1 }\end{array}$} & \multicolumn{2}{|c|}{ Before treatment } & \multicolumn{2}{|c|}{ After treatment } \\
\hline & & & & NC1 & COL7 & NC1 & COL7 \\
\hline 1 & Female & 16 & c. $682+1 G>A / c .5532+6 T>C$ & - & - & - & + \\
\hline 2 & Female & 18 & c.425A>G(p.Lys142Arg)/c.425A>G(p.Lys142Arg) & - & - & - & + \\
\hline 3 & Female & 28 & c.425A>G(p.Lys142Arg)/c.5884A>C(p.Thr1962Pro) & - & + & + & ++ \\
\hline 4 & Female & 42 & c.425A>G(p.Lys142Arg)/c.7904G>A(p.Gly2635Asp) & + & + & ++ & + \\
\hline 5 & NHS & 26 & & +++ & +++ & +++ & +++ \\
\hline
\end{tabular}

NHS - normal human skin, RDEB - recessive dystrophic epidermolysis bullosa, NC1-monoclonal antibody (red) Col7-polyclonal antibody (green).

tration of the B1 gentamicin component should be determined for patients with EB. That was not performed before, either for our research or previously published papers. In the future we are planning to research and determine the gentamicin concentration with a larger research group.

At this stage, when the patients used mouth rinse with gentamicin $0.3 \%$ solution, the probability of its absorption and impact on the general health seemed low, even with ingestion of traces of the drug. We were al- ways mindful of reported adverse reactions to general gentamicin therapy. We have not noted any side effects in our patients during the entire time of experiment. The trials to treat skin erosions topically with gentamicin in patients with RDEB have been discussed already in the literature. Undoubtedly, the high safety profile of local gentamicin therapy of skin erosions is an advantage in comparison to systemic therapy. Gentamicin is poorly absorbed when administered orally, which is also an advantage for topical treatment of mucosal erosions in patients 
with EB $[13,14]$. In the future a higher and strictly recognized concentration of B1 component of gentamycin and more adhesive formulas, like dental adhesive pastes, should be taken to consideration. It may improve the local absorption of active components of the drug without the fear of overdosage and appearance of side effect concerns with chronic use of gentamycin.

To the best of our knowledge, this is the first study on efficacy of topical gentamicin treatment of mucosal erosions in the course of EB. In our study 4 female patients with recessive dystrophic EB-severe generalized (RDEBsev gen) RDEB and extensive oral erosions treated with gentamycin $0.3 \%$ solution for 4 weeks showed a clinical improvement in healing oral wounds.

Patients with RDEB-sev gen showed the highest mean of mucosal sites involved by the lesions. The interobserver reliability on the total of lesions was excellent for only 3 sites: lower lip, hard palate and tongue [7] and it was the main limitation to estimate the number of new blister and erosions formation as well as calculation of the involved mucosal surface area in our study. Despite the above-mentioned difficulties in our study it was possible to observe the significant reduction in new blister formation and mucosal surface area of erosions and gingivitis suggesting proper wound healing. All the patients reported a significant reduction in pain and gingival bleeding which allowed better dental care.

Wright et al. have shown that the prevalence of caries was significantly higher in EB individuals than among healthy people. It has been hypothesized that excessive caries is a result of the presence and severity of the soft tissue involvement, which leads to alteration in diet, increased oral clearance time, creation of an abnormal tooth/soft tissue relationship and prevention of normal oral hygiene measures [15]. For this reason hygiene is essential in patients with EB.

Interestingly, in our cohort of RDEB patients we found approximately two-fold reduction in the Plaque Index, which indicated better oral hygiene during the treatment. The improvement of mucosal lesions reduced the risk of complications of tooth decay, such as pulpitis or even premature teeth loss and avoided endodontic treatment - so difficult in these patients. The tooth loss in patients with $E B$ is a significant clinical problem impeding the proper crushing of the food bite. As a consequence, nutritional disorders and more frequent damage to the oral mucosa, throat and oesophagus may occur. The performance of removable prosthetic restorations in these cases is fraught with numerous complications and it is necessary to consider micro-invasive implantological methods [16].

It should not be forgotten that all problems mentioned above and properly functioning oral system also enables the correct speaking and food chewing, which reduces the risk of damage to mucous membranes of the digestive system and malnutrition, which, in turn, has an impact on the local condition of the skin and healing of skin wounds [3].

Clinical improvement of the oral lesions during gentamicin treatment depended, at least in part, on the re-expression of type VII collagen; of note in all studied patients partial re-expression of type VII collagen was observed.

Patients included in the study had well-defined mutations of the COL7A1 gene which are known to introduce a premature termination codon (PTC) due to splicing aberration. Two patients (no. 1 and 2 in Table 1) represent the most severe variant of RDEB - the so-called RDEB-sev gen (formerly known as. Hallopeau-Siemens). Patient no. 1 represents heterozygous mutation $\mathrm{c} .682+1 \mathrm{G}>\mathrm{A} / \mathrm{c} .5532+6 \mathrm{~T}>\mathrm{C}$ and patient no. 2 has homozygous mutation (c.425A>G) in exon 3 (the most common in Poland) resulting in aberrant splicing of type VII collagen at the early stage of its synthesis, which explains the clinical severity and absence of type VII collagen expression before the treatment [17]. In both RDEB-sev gen patients, extensive oral wounds were accompanied by pseudo-syndactyly and changes in internal organs $[13,14]$. After the gentamicin treatment, the immunofluorescence study revealed partial re-expression of type VII collagen in the patients' skin.

In the two nHS-RDEB patients, laboratory tests performed prior to the treatment showed traces of type VII collagen and an increased expression of collagen type VII after therapy. Two remaining patients (no. 3 and 4) had compound heterozygous mutation (c.425A $>\mathrm{G}$ ) accompanying "milder" mutations (c.5884A>C and c.7904G>A, respectively).

Despite the fact that gentamicin $0.3 \%$ solution mouth rinse can prevent mucosal injuries, everyday use of gentamicin may result in the resistance to this antibiotic, therefore this treatment could be recommended for EB patients with very severe lesions and for a relatively short period of time. Moreover, treatment of the oral cavity is necessary since all sharp edges irritate the mucosa, which results in fibrosis of the tissue. The topical use of gentamicin in the form of a rinse may be considered as a prophylaxis of dissemination of mucosal lesions resulting from the invasive nature of planned dental, laryngological or endoscopic procedures. We also consider ingestion of this solution by patients and testing its efficacy on the oral and oesophageal wounds, but it requires further research and observation. Mucosal lesions are an important dental and therapeutic problem. There is a need to intensify activities to increase health awareness in order to improve the functioning of the first section of the gastrointestinal tract, i.e. the oral cavity. General treatment of the disease is dealt with first of all by a dermatologist. The dentist's role is to perform hygiene procedures, sanitation of the oral cavity and to eliminate irritating factors such as sharp edges teeth. Such activities will allow for better nutrition, and thus better general health condition. 


\section{Conclusions}

After a 4-week use of gentamycin $0.3 \%$ solution for oral cavity twice a day in the patients with RDEB showing various mutations introducing PTC in the gene encoding collagen type VII, a clinical improvement was observed, namely oral wounds healing and becoming less painful, moreover the immunofluorescence study of oral samples showed partial re-expression of collagen type VII.

\section{Acknowledgments}

This work was performed as part of the programme of the National Centre for Research and Development, Poland, STRATEGMED2/269807/NCBR2015/acronym: BIOOPA

\section{Conflict of interest}

The authors declare no conflict of interest.

\section{References}

1. Lee JYW, Liu L, Hsu CK, et al. Mutations in KLHL24 add to the molecular heterogeneity of epidermolysis bullosa simplex. J Invest Dermatol 2017; 137: 1378-80.

2. Dundappa Parushetti A, Agrawal JM, Nanjannawar LG, Agrawal MS. Oral manifestations of epidermolysis bullosa dystrophica: a rare genetic disease. BMJ Case Rep 2013; 2013: bcr2012007963.

3. Woodley DT, Cogan J, Hou Y, et al. Gentamicin induces functional type VII collagen in recessive dystrophic epidermolysis bullosa patients. J Clin Invest 2017; 127: 3028-38.

4. Fortuna G, Aria M, Cepeda-Valdes R, et al. Clinical features of gingival lesions in patients with dystrophic epidermolysis bullosa: a cross-sectional study. Australian Dental J 2015; 60: 18-23.

5. Wright JT. Oral manifestations in the epidermolysis bullosa spectrum. Dermatol Clin 2010; 28: 159-64.

6. Murrell DF, Pasmooij AMG, Pas HH, et al. Retrospective diagnosis of fatal bp180-deficient non-herlitz junctional epidermolysis bullosa suggested by immunofluorescence (IF) antigen-mapping of parental carriers bearing enamel defects. J Investig Dermatol 2007; 127: 1772-5.

7. Fortuna G, Lozada-Nur F, Pollio A, et al. Patterns of oral mucosa lesions in patients with epidermolysis bullosa: comparison and agreement between oral medicine and dermatology. J Oral Pathol Med 2013; 10: 733-40.

8. Chaffee BW, Feldens CA, Rodrigues PH, Vítolo MR. Feeding practices in infancy associated with caries incidence in early childhood. Community Dent Oral Epidemiol 2015; 43: 338-48.

9. Pizzichini M, Pandolfi ML, Arezzini L, et al. Labelling of uric acid and allantoin in different purine organs and urine of the rat. Life Sci 1996; 59: 893-9.

10. Nystrom A, Thriene K, Mittapalli V, et al. Losartan ameliorates dystrophic epidermolysis bullosa and uncovers new disease mechanisms. EMBO Mol Med 2015; 7: 1211-28.

11. Snauwaert JJL, Yuen WY, Jonkman MF, et al. Burden of itch in epidermolysis bullosa. Br J Dermatol 2014; 171: 73-8.

12. Hubbard LD, Mayre-Chilton K. Quality of life among adults with epidermolysis bullosa living with a gastrostomy tube since childhood. Qual Health Res 2015; 25: 310-9.

13. Bruckner-Tuderman L. Can type VII collagen injections cure dystrophic epidermolysis bullosa? Mol Ther 2009; 17: 6-7.
14. Baradaran-Heravi A, Niesser J, Balgi AD, et al. Gentamicin B1 is a minor gentamicin component with major nonsense mutation suppression activity. Proc Natl Acad Sci USA 2017; 114: 3479-84.

15. Wright JT, Fine JD, Johnson L. Dental caries risk in hereditary epidermolysis bullosa. Pediatr Dent 1994; 16: 427-32.

16. Wychowański PS, Woliński J, Kacprzak MT, et al. Immediate palatal molar implants: a simple, safe, minimally invasive technique. Int J Period Restor Dent 2017; 37: 297-301.

17. Wertheim-Tysarowska K, Sobczyńska-Tomaszewska A, Kowalewski C, et al. Novel and recurrent COL7A1 mutation in a Polish population. Eur J Dermatol 2012; 22: 23-8. 